\title{
LA PESCA DEL CANGREJO ROJO AMERICANO Y SU INFLUENCIA EN EL ENTORNO DEL PARQUE DE DOÑANA
}

\author{
Fernando MOLINA VAZQUEZ *
}

\section{INTRODUCCION}

Las marismas del Guadalquivir, y el mismo río, han representado desde tiempos históricos un potencial pesquero que ha servido de sustento a numerosas familias. Sin embargo, y pese a la amplitud del tema, no existe una verdadera tradición bibliográfica sobre el particular. Podemos considerar como estudios base del encuadre temático los realizados por MUÑOZ PEREZ (1972), en el que se aborda la pesca a nivel comarcal desde el punto de vista geográfico-histórico, y el de SUAREZ JAPON (1978), centrado en torno al municipio de Coria del Río. Pueden citarse otros estudios aislados y puntuales como los realizados por GUTIERREZ RODRIGUEZ (1962), sobre el esturión en el río Guadalquivir, o el de LIBRERO RUIZ (1980), sobre el cangrejo rojo y su pesca.

Desde 1980 venimos interesándonos por la problemática del sector cangrejero de las marismas del Guadalquivir (ROBLES MOLINA y col., 1982) dado el auge de esta actividad, y por su impacto medioambiental (MOLINA, 1984)**.

\section{ZONIFICACION PESQUERA DEL BAJO GUADALQUIVIR}

El espacio hidrológico del tramo final del Guadalquivir a efectos de biomasa queda escuetamente delimitado en tres grandes zonas: cauce pre-estuario, estuario y marismas (RIBERA DE VANNEY, 1970).

* Biólogo. Agencia de Medio Ambiente. Junta de Andalucía

* Este trabajo tiene su inicio en la ponencia que desarrollamos en las I Jornadas de Investigaciones en Doñana, en Junio de 1984. Los datos analíticos surgieron de una investigación realizada para el ICONA (1983), del seguimiento de la pesca del cangrejo en ese año. Agradecemos a ese Instituto, y al biólogo D. Juan Manuel de Benito Ontañón el interés por la dirección del trabajo. Asimismo, al ingeniero de montes D. José Antonio Robles Clavijo, por sus indicaciones en el desarrollo del trabajo del cangrejo en la marisma, al Dr. D. Fernando Díaz del olmo por la lectura de este artículo, y al Dr. D. Tomás Azcárate y Bang, Director de la Agencia de Medio Ambiente, por sus indicaciones. 
Esta zonificación es el hábitat de un conjunto de especies de alta variedad e interés piscícola, que se han visto afectadas en los últimos decenios, tanto por los impactos degradantes de los residuos orgánicos e inorgánicos que al río se han venido vertiendo, como por las distintas manipulaciones antrópicas que ha sufrido (MENANTEAU, 1984).

El cauce pre-estuario ha representado una excelente área de pesca, y se ha pasado de una situación desahogada, a una crítica próxima a la extinción. El inventario piscícola de este tramo ha sido amplio (LOZANO REY, 1947), pero no obstante el aprovechamiento comercial se basaba en la captura de la angula, albur, barbo, camarón y el esturión. La angula se viene pescando en las orillas del Guadalquivir con artes tradicionales, esperando su habitual paso nocturno en cordón por las márgenes. Su comercialización normalmente era fuera de nuestra región debido a la falta de hábito de consumo de esta especie, que por otro lado son las denominadas de "lomo blanco", de inferior calidad. El albur, mugílido eurihalino de gran adaptabilidad a las distintas concentraciones salinas, ha sido bastante capturado a lo largo del río siendo objeto de un comercio local, sobre todo en las localidades de Coria del Río y Alcalá del Río, comercio que aún persiste. Otra especie de interés es el camarón, crustáceo muy apreciado en todo el Bajo Guadalquivir y que se pesca por medio de barcas provistas de unas redes especiales denominadas "camaroneras". Sin embargo, una de las especies de más valor en este entorno ha sido el esturión, abundante en otra época, que remontaba el río para desovar. Era aprovechado en su paso para capturarlo, existiendo, en 1932 una empresa ubicada en Coria del Río, de la sociedad Ybarra, que se dedicaba a la transformación en caviar y su posterior comercialización.

El estuario supone uno de los hábitats de mayor diversidad ecológica, concentrándose en él las especies de mayor valor comercial. Por medio de diferentes artes y técnicas de pesca se ha venido capturando especies como la dorada, lubina, lisa, lenguado, langostino y baila. Actualmente sigue suponiendo una buena base de la actividad pesquera local de poblaciones como Sanlúcar de Barrameda.

En las marismas transformadas para la extracción de sal, salinas, se viene, aún hoy en día, manteniendo la pesca de estero, modalidad ésta que supone una actividad complementaria importante a la de la sal. Se capturan prácticamente las mismas especies que en la desembocadura, pero con un valor comercial más alto.

Ya en las marismas, los sistemas de pesca y especies capturadas varían. En los caños, brazos, arroyos y lucios se capturaba la anguila y la carpa como especies sustentadoras del reducido sector de pescadores, asentados en los pequeños y nuevos poblados marismeños. No obstante, la explotación de la anguila de forma profesional es relativamente reciente, surgiendo en los años 50 , debido al interés mostrado por algunos exportadores de origen valenciano y que descubrieron el gran potencial piscícola de la zona. 
El aprovechamiento pesquero se realizaba por medio de concesiones establecidas por las Comunidades de Regantes a los compradores de anguilas, que a su vez éstos compraban el producto a cuadrillas creadas al efecto. No es hasta 1967 cuando estas cuadrillas se unen formando la denominada "Agrupación Sindical Profesional de pescadores del Bajo Guadalquivir y sus Marismas", habitantes de los pueblos contiguos y que se dedicaban anteriormente a la caza de patos o recolección de la enea. En 1969 obtuvieron la concesión para el aprovechamiento industrial de diez cotos de pesca.

\section{UNA NUEVA ALTERNATIVA: LA PESCA DEL CANGREJO ROJO}

El cangrejo rojo de las marismas (Procambarus clarkii, Girard), denominado en su lugar de origen, Louisiana-Estados Unidos, como Red swamp crawfisch (cangrejo rojo de pantano), se introdujo en nuestras marismas en Mayo de 1969 (ALGARIN, 1980), mediante siembras realizadas en una finca de Puebla del Río, de una partida procedente de Monroe (Louisiana). Desde esta fecha ha ido invadiendo paulatinamente las zonas de marismas, hasta llegar, en el año 1974, a su expansión máxima, distribución que permanece prácticamente imperturbable hasta nuestros días.

La rápida proliferación de este astácido por el ámbito marismeño se ha debido a la gran accesibilidad encontrada, ya que el escalón trófico que ha ocupado estaba vacío y no existían predadores de importancia. Esto, complementado con el manejo de las aguas para el cultivo del arroz, supuso para el cangrejo un hábitat mucho más idóneo que el ocupado en los pantanos de su lugar de origen.

La introducción se efectuó de forma experimental, con el beneplácito de la Administración, y con ella se pretendía el cultivo de esta especie de forma extensiva en la cautividad. Su lugar de suelta fue en la finca Casablanca, dedicada al cultivo del arroz, y que era regada mediante bombeo de las aguas de río Pineda, aguas que vuelven a su cauce una vez utilizadas. Así el cangrejo pudo fácilmente utilizar esta vía para su distribución por las fincas colindantes, siendo de esta manera como, a finales de 1975 ya había colonizado de forma natural todo el área del río Pineda hasta el Guadalquivir.

A partir de 1975, la expansión natural es complementada con la ejercida por los propios pescadores, distribuyendo el cangrejo por todas las marismas, brazos y caños de las dos orillas del Guadalquivir.

Esta distribución desmesurada de esta especie hace cambiar la actividad pesquera de los profesionales de la marisma. La Agrupación de Pescadores antes citada empieza, en 1976, la pesca del crustáceo, simultaneándola con la de la anguila y realizándola en los cotos industriales de pesca que tenían concedidos, pero es en 1978, con la anulación de estos cotos cuando empieza una nueva etapa. La Agrupación cambia de actividad y de nombre comercial, 
pasando a denominarse "Pescadores del Bajo Guadalquivir y sus marismas, S. Cop. L.". Esta nueva etapa da paso a un aprovechamiento incontrolado de este recurso, determinando un crecimiento en número de pescadores y centros astacícolas. Se pasa de una sola Cooperativa con 43 miembros en 1968 , a 400 pescadores profesionales contabilizados, tanto en dedicación exclusiva como parcial, y nueve centros depuradores, en 1981.

Hasta 1979, todos los pescadores pertenecían a la antigua Agrupación Sindical. Es a partir de esta fecha cuando aparecen gran número de nuevos pescadores, algunos con dedicación exclusiva, y la mayoría por temporadas. La Agrupación pasa de 48 en esta fecha a 136 en 1981.

La evolución del número de asociados puede constatarse en la figura adjunta (ROBLES, MOLINA y col. 1982).

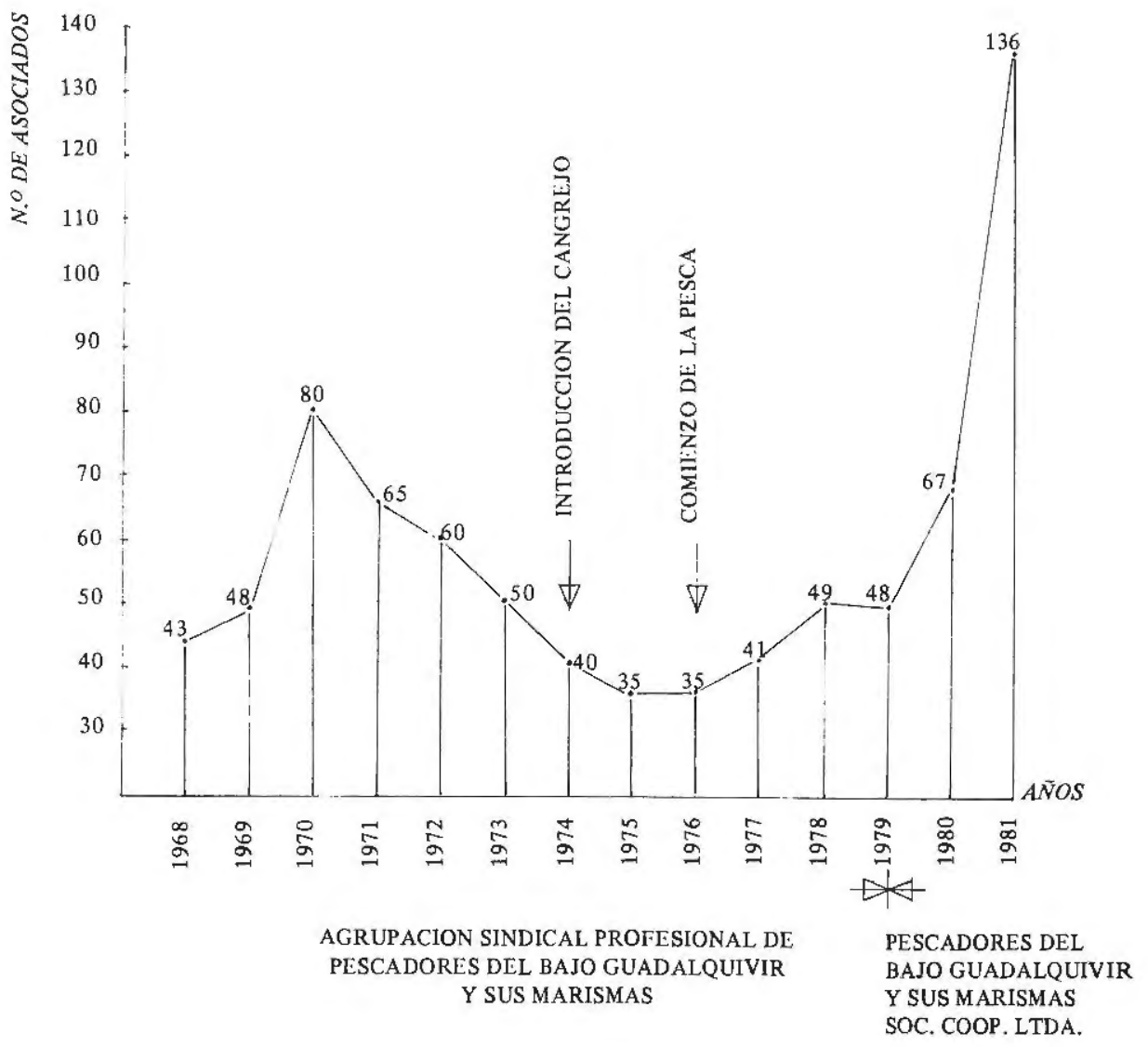


El sistema de pesca ha ido evolucionando rápidamente en los últimos años. Su explotación se realiza mayoritariamente en los campos de arroz, que, debido a las técnicas de riego de estos cultivos permite tener inundada grandes extensiones de la marisma aquellas épocas en que por efectos naturales permanecería seca, además de mantener con agua los caños, acequias que sirven de infraestructura a este cultivo.

Una idea de las amplias posibilidades de expansión que ha tenido el cangrejo nos la da la superficie que anualmente se inunda con fines Piscícolas, que en 1981 ascendió a 27.700 Ha. (datos de la Federación de Agricultores Arroceros), distribuída en ambas márgenes del Guadalquivir.

El cangrejo era pescado al principio tanto en las tablas de arroz como en los caños y brazos y se utilizaba a tal efecto las "nasas" (redes cilíndricas de malla con una luz de uno o dos centímetros y varias muertes), artes de pesca utilizadas para la captura de la anguila. Cada pescador tenía un número variable de estas redes y eran revisadas cada cierto tiempo, oscilable éste según la época del año, y vendía las capturas a los centros depuradores, dos al principio, las cuales tenían un precio que variaba diariamente conforme a la oferta y la demanda. Más tarde este sistema de pesca varió, confeccionándose redes especiales al efecto, que diferían de las anteriores particularmente en el tamaño, y se denominaron "nasas cangrejeras". Estas se colocaban en los caños, tanto naturales como del arroz, situando la boca de entrada cerca de la orilla y con una ligera inclinación con respecto a ésta de unos $45^{\circ}$.

La proliferación de pescadores y nasas ha sido progresiva con las del cangrejo. En el cuadro I se puede apreciar el aumento vertiginoso de las capturas controladas, es decir, del stock contabilizado por los centros depuradores.

CUADRO I

\begin{tabular}{|c|c|c|}
\hline Año & & $\begin{array}{l}\text { Kgs, de cangrejo } \\
\text { comercializados }\end{array}$ \\
\hline 1976 & $\ldots \ldots \ldots \ldots$ & 5.000 \\
\hline 1977 & $\ldots \ldots \ldots \ldots \ldots$ & 15.000 \\
\hline 1978 & $\ldots \ldots \ldots \ldots$ & 50.000 \\
\hline 1979 & $\ldots \ldots \ldots \ldots \ldots$ & 150.000 \\
\hline 1980 & $\ldots \ldots \ldots \ldots$ & 510.000 \\
\hline 1981 & $\ldots \ldots \ldots \ldots$ & 1.700 .000 \\
\hline 1982 & $\ldots \ldots \ldots \ldots$ & 3.000 .000 \\
\hline
\end{tabular}


En el año 1983, hubo una disminución drástica en el total de capturas, debido a la sequía padecida en la zona y que dió lugar a una falta de agua para el riego del arroz.

El aumento progresivo de capturas fue inversamente proporcional al precio por $\mathrm{kg}$. De pagarse a $800-900 \mathrm{pta} / \mathrm{kg}$. al principio del boom del cangrejo, precio gustosamente pagado en los lugares consumidores del cangrejo de río español que pagaban hasta $3.000 \mathrm{pta} / \mathrm{kg}$., a $150 \mathrm{pta} / \mathrm{kg}$. en temporadas bajas del año 1982 y $350 \mathrm{pta} / \mathrm{kg}$. en las altas de ese mismo año.

Pero no todo fueron parabienes con el resurgimiento de este nuevo recurso. La proliferación de las poblaciones de cangrejos supuso una verdadera plaga para el cultivo del arroz, porque por su lado, las tablas de cultivo eran invadidas continuamente por los pescadores con el consiguiente deterioro de las plantaciones tanto por el trasiego de personas como por la colocación de las artes de pesca, unido al daño ocasionado por los "domingueros", por lo general de mayor repercusión, y por otro lado, los daños ocasionados al horadar con las quelas el terreno en las épocas de falta de agua o de cría, formando así las llamadas madrigueras o torretas, algunas de casi medio metro. Las numerosas perforaciones de caños y tablas produce efectos de desmoronamiento de almorrones, vaciado de los campos, así como alteraciones en los sistemas de riegos. Estos daños han sido valorados por los arroceros como graves, surgiendo así un enfrentamiento entre agricultores y pescadores.

\section{INFLUENCIA DE LA PESCA EN DOÑANA}

La expansión incontrolada del cangrejo no respetó los límites naturales del Parque Nacional. Su presencia se hace patente en 1975, tanto en el arroyo de la Rocina como en el caño de las Nuevas. La población evolucionó con normalidad, y no es hasta 1978 , que, debido a una siembra furtiva en el área del arroyo de la Rocina, cuando, y a raíz de este hecho, se dispara la población. Ya en 1979, surge el aprovechamiento pesquero dentro del Parque, relegándose esta actividad a los meses comprendidos entre Marzo y Junio, y dependiendo este intervalo tanto de la pluviometría como de las temperaturas.

La importancia de la pesca en las marismas y caños dulces del Parque Nacional de Doñana se debe a dos razones principales:

1. Debido al manejo del cultivo del arroz, así como de la ínfima superficie de marisma que queda anegada por efectos pluviales durante el comienzo del ciclo biológico activo del cangrejo, éste se desarrolla tan sólo en aquellas zonas que mantienen un nivel aceptable de agua, que, en la zona arrocera, se reduce a los caños, y en el Parque, a las marismas naturales del área de desbordamiento de la Rocina y de la 
Madre de las Marismas. Por ello, el desarrollo óptimo de poblaciones comerciales de cangrejos durante los meses de Marzo a Mayo se da casi exclusivamente en terrenos del Parque, constituyendo estos los únicos cotos de pesca en estas fechas.

2. El cangrejo del Parque tiene un ciclo biológico distinto al que habita en el resto de la zona cangrejera, caracterizándose por su mayor tamaño y su superior calidad gastronómica. Esto, junto a la escasez de cangrejos al principio de temporada, hace que alcance un máximo de precio en la fluctuación anual.

A no ser por estas dos razones, la pesca en el interior del Parque perdería su importancia, ya que el volumen de capturas representa tan sólo el $3 \%$ de las totales (datos referidos a las campañas de 1981 y 82).

Desde la aparición del cangrejo como recurso en el área que nos ocupa ha levantado una serie de polémicas sobre la cuestión jurídica del aprovechamiento (ROBLES, J.A., MOLINA, F., ALGARIN, S., y otros, 1982) llegándose a pescar incontroladamente durante las campañas de 1979 y 1980, no siendo así en las sucesivas de 1981, 82 y 83, mediante acuerdos de la Administración con las Cooperativas.

En 1982 se hizo una evaluación de los efectos producidos por la pesca durante la campaña, realizándose en los meses de marzo, abril, mayo y junio, arrojando unas cifras alarmantes con respecto al impacto producido sobre la avifauna acuática (CORONADO, R., 1982, Informe al Patronato). Estos indicaban la captura de un ave por cada 5,4 nasas/día. Las especies capturadas se centraban en 12 especies, de las que podemos resaltar el calamón, el pato colorado y la cerceta carretona.

Para la campaña de 1983 se pensó en un principio en la prohibición de la pesca, dado el alarmante índice de capturas obtenido en la anterior, pero se llegó a un acuerdo con la Federación de Pescadores para autorizar la pesca en el período anterior a la nidificación de las aves acuáticas, período que quedó establecido desde el 8 de Marzo al 15 de Abril, con una serie de condiciones como:

- Zona autorizada de pesca. Esta correspondía al área de la Madre de las Marismas, desde el puente de la Canariega hasta la recta que une la "Casa de los Guardas" con la llamada "Cancela de las Escupideras", situada en el muro que es límite del Parque Nacional. Limitaba un pequeño sector en el Soto Chico, debido a la nidificación del águila imperial.

- Horario de supervisión de las redes. Se estableció entre las 7 y las 11 de la mañana. 
- Cupo de pescadores. Máximo 50 diarios.

- Cupo de artes de pesca. El total de las nasas colocadas fue de 2.160.

- Reducción obligatoria de la altura de boca de las nasas, mediante ] colocación de una red vertical.

- Seguimiento por parte de ICONA de los efectos producidos por dich pesca.

Como resultados del seguimiento efectuado sobre la pesca en ese año s obtuvieron los datos que se muestran en los cuadros II y III. Estas fueron le capturas estimadas durante el período de pesca, tanto de cangrejos como d otras especies.

Durante la campaña 1984, la pesca fue prohibida en todo el ámbito dt Parque y Pre-Parque, originándose enfrentamientos entre la Administració del Parque y la Federación de pescadores, que, a pesar de la prohibiciór colocaron nasas en terrenos del Parque, dando como resultado la incautació: de más de 2.000 nasas durante los meses de actividad cangrejera.

Para años sucesivos, se hace inminente el acondicionamiento de zona marismeñas inundadas al efecto, y fuera de los límites de influencia del Par que, y su posterior concesión a la Federación de Pescadores, evitando asíl invasión de Doñana en los meses de inactividad cangrejera en el resto d la marisma. Una de las áreas alternativas y con mayores posibilidades sería 1 del Plan Almonte-Marisma, colindante con el Parque.

El impacto producido por el cangrejo en los ecosistemas del Parqur Nacional podemos valorarlos desde dos perspectivas:

1. Por un lado, la introducción de una especie alóctona ha producidc variaciones en las cadenas tróficas, siendo pronto para valorar el po sible desequilibrio ecológico de las comunidades animales y vegetales Especies como la cigüeña, la garza, la focha, han desviado sus hábito: alimenticios hacia esa fácil y abundante presa que supone el cangrejo Este, por otro lado, incide notablemente sobre las puestas de anfi bios, peces, y sobre la vegetación subacuática.

2. Otra vertiente a evaluar es la incidencia directa de los pescadores so bre las áreas húmedas. Normalmente la época de pesca en la Rocina lugar que cría los mejores ejemplares de cangrejos, coincide con la puesta de una amplia gama de especies de aves acuáticas. El trasiegc de un elevado número de pescadores entre los caños y áreas inunda. das del entorno causa una considerable molestia que influye directa. mente sobre los resultados de la puesta. A esto se une el efecto de las nasas, ya que muchas de ellas presentan un blanco fácil para patos y zampullines, que una vez introducidos por la boca de la red es difícil que escapen, pereciendo normalmente ahogados. 
CUADRO II

\begin{tabular}{|c|c|c|c|c|c|c|c|c|c|c|c|c|c|}
\hline \multirow{2}{*}{ Semanas } & \multicolumn{2}{|c|}{ Invertebrados } & \multicolumn{4}{|c|}{ Anfibios } & \multicolumn{3}{|c|}{ Reptiles } & \multicolumn{2}{|c|}{ Peces } & \multicolumn{2}{|c|}{ Aves } \\
\hline & $\begin{array}{l}\text { Ditiscus } \\
\text { Cybister }\end{array}$ & $\begin{array}{l}\text { Hidrous } \\
\text { Pictus }\end{array}$ & $\begin{array}{l}\text { Pleurodeles } \\
\text { Walu }\end{array}$ & $\begin{array}{l}\text { Pelobates } \\
\text { Cultripes }\end{array}$ & $\begin{array}{l}\text { Diseoglosus } \\
\text { Pietus }\end{array}$ & $\begin{array}{c}\text { Rana } \\
\text { Ridibunda }\end{array}$ & $\begin{array}{c}\text { Mauremys } \\
\text { Caspica }\end{array}$ & $\begin{array}{c}\text { Natrix } \\
\text { spp. }\end{array}$ & $\begin{array}{c}\text { Blanus } \\
\text { Cinereus }\end{array}$ & $\begin{array}{l}\text { Anguilla } \\
\text { Anguille }\end{array}$ & $\begin{array}{c}\text { Cyprinus } \\
\text { Carpio }\end{array}$ & $\begin{array}{l}\text { Anes } \\
\text { Plat. }\end{array}$ & $\begin{array}{l}\text { Limosa } \\
\text { Limoss }\end{array}$ \\
\hline Primera & 9.682 & 826 & 835 & 51 & 7 & 29 & 21 & 7 & 14 & 848 & 7 & - & - \\
\hline Segunda & 5.509 & 629 & 42 & 122 & - & 24 & 6 & - & - & 85 & - & - & - \\
\hline Tercera & 9.233 & 2.140 & 172 & 356 & - & 21 & - & 10 & - & 64 & - & - & - \\
\hline Cuarta & B.000 & B20 & 27 & 75 & - & - & - & - & - & 82 & 6 & 10 & 6 \\
\hline Quinta & 4.082 & 141 & 4 & 80 & - & 35 & 4 & 一 & - & 8 & - & - & - \\
\hline TOTAL & 42.770 & 5.174 & $1,25 B$ & 785 & 10 & 128 & 36 & 19 & 16 & 1.260 & 13 & 10 & 6 \\
\hline
\end{tabular}


CUADRO III

\begin{tabular}{lrrr}
\hline Semanas & $\begin{array}{c}\text { Peso (Kg.) } \\
\text { No Comercial }\end{array}$ & $\begin{array}{c}\text { Peso (Kg.) } \\
\text { Comercial }\end{array}$ & $\begin{array}{c}\text { Peso (K.) } \\
\text { Total }\end{array}$ \\
\hline Primera & & & \\
Segunda & 855 & 1.020 & 1.875 \\
Tercera & 846 & 1.399 & 2.245 \\
Cuarta & 1.280 & 991 & 2.271 \\
Quinta & 1.404 & 1.363 & 2.767 \\
Total & 914 & 1.416 & 2.330 \\
\hline
\end{tabular}

La prohibición de la pesca en el entorno del Parque Nacional no es un mero capricho. Al margen de representar un recurso aprovechable, hay que recordar el objetivo principal por el que se creó el Parque: la conservación de la flora, fauna y gea, supeditándose todo tipo de aprovechamiento a esta premisa.

\section{BIBLIOGRAFIA CITADA}

ALGARIN VELEZ, S. (1980): "Problemática y perspectivas de la introducción del cangrejo". Jornadas de estudio del cangrejo. Junta de Andalucía, pp. 25-31.

LIBRERO RUIZ, M. (1980): "Biología y pesca del cangrejo". Jornadas de estudio del cangrejo. Junta de Andalucía, pp. 17-25.

LOZANO REY, L. (1952): "Los peces fluviales de España”. Pub. M. ${ }^{\circ}$ de Agricultura. Madrid.

MEN AN TEAU, L. (1984): "Evolución histórica y consecuencias morfológicas de la intervención humana en las zonas húmedas: el caso de las marismas del Guadalquivir'. Las zonas húmedas andaluzas. MOPU, pp. 43-76.

MOLINA VAZQUEZ, F. (1984): "Impacto de la pesca del cangrejo rojo (Procambarus clarkii), en los ecosistemas marismeños del Parque Nacional de Doñana, durante la campaña de 1983". I Jornadas de investigación en Doñama. Junio, 1984.

MUÑOZ PEREZ, J. (1972): "La pesca en la desembocadura del Guadalquivir". Instituto de Estudios Gaditanos. Diputación Provincial. Cădiz.

RIBERA DE VANNEY, J. (1970): "L'Hidrologie du Bas Guadalquivir". C.S.1.C. Madrid.

ROBLES, J.A., MOLINA, F., y col. (1982): "Estudio sobre el cangrejo rojo americano en las marismas del Guadalquivir". ICONA. (Inédito).

SUAREZ JAPON, M. (1970): "La pesca en el Eajo Guadalquivir: hacia el final de una actividad" GADES, pp. 163-182. 